\title{
Endoscopic ultrasound-guided fine-needle aspiration of parathyroid adenoma in a patient with hypercalcemia and ureter stone
}

\author{
Kwangwoo Nam ${ }^{1}$, Sungwook $\mathrm{Jang}^{1}$, Sung Hyeok Ryou ${ }^{1}$, Kyungjun Kim ${ }^{1}$, and Yong-Moon Lee ${ }^{2}$
}

Departments of ${ }^{1}$ Internal Medicine and ${ }^{2}$ Pathology, Dankook University Hospital, Cheonan, Korea
Received: June 2, 2020

Revised : June 8, 2020

Accepted: June 8, 2020

\section{Correspondence to}

Kwangwoo Nam, M.D.

Tel: +82-41-550-3092

Fax: +82-41-550-7050

E-mail: nambag1108@gmail.com https://orcid.org/0000-00033720-9820
A 61-year-old man visited our hospital with lower abdominal pain. He had a history of a ureter stone and had underwent ureteroscopic stone removal 1 month previously. Colonoscopy and abdominopelvic computed tomography showed prominent wall thickening with significant stricture in the sigmoid colon. Repeated forceps biopsies showed no malignant cells. As the possibility of submucosal infiltrating type cancer could not be excluded, surgical resection was considered. However, laboratory tests showed hypercalcemia (11.5 mg/dL), elevated ionized calcium $(1.40 \mathrm{mmol} / \mathrm{L})$, and elevated intact parathyroid hormone (PTH) levels $(97.1 \mathrm{pg} / \mathrm{mL})$. Due to the suspicion of primary parathyroidism, chest computed tomography was performed; it revealed a 3-cm-sized highly enhanced mass in the right upper tracheal area
(Fig. 1A). Neck ultrasound-guided fine-needle aspiration (US-FNA) failed to target this mass; hence, endoscopic ultrasound-guided fine-needle aspiration (EUS-FNA) was performed. In the upper esophagus, $22 \mathrm{~cm}$ from the upper incisor, EUS showed a $2.6 \times 1.8-\mathrm{cm}-$ sized heterogenous hypoechoic mass with a focal anechoic cystic lesion (Fig. 1B). Using a 22-gauge needle, EUS-FNA was performed without immediate complications, revealing several epithelial cell clusters without nuclear atypia, suggestive of parathyroid adenoma (H\&E, ×400) (Fig. 2A). ${ }^{99 m}$ Technetium methoxyisobutylisonitrile (MIBI) single-photon emission computed tomography (SPECT) showed delayed washout at the postero-inferior aspect of the lower pole of the right thyroid lobe. Following treatment of hypercalcemia with hydration, he successfully under-
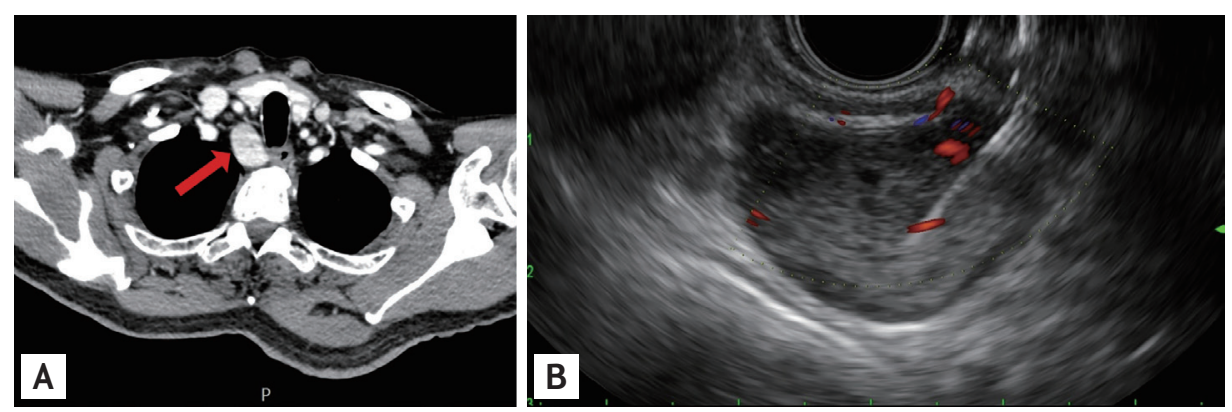

Figure 1. (A) Chest computed tomography revealed $3 \mathrm{~cm}$ sized oval shaped well-enhancing mass in the right upper tracheal area (arrow). (B) Endoscopic ultrasound-guided fine-needle aspiration was performed to confirm the diagnosis. 

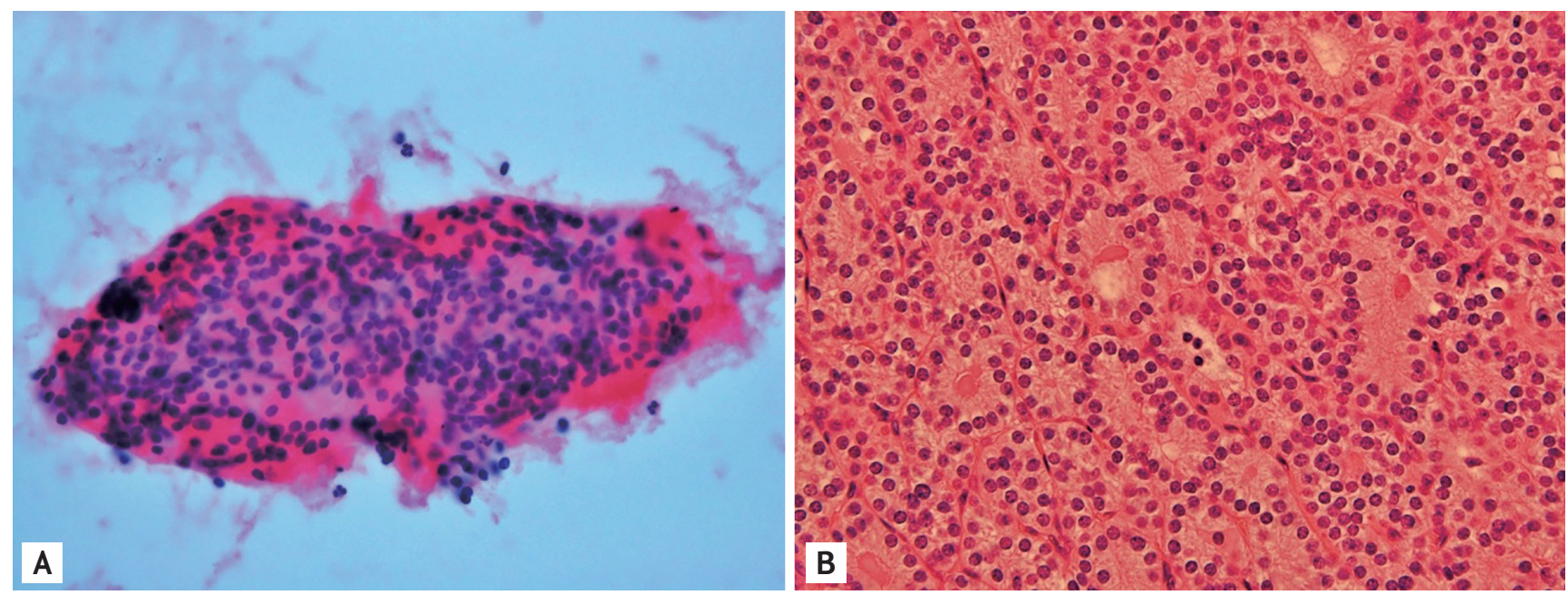

Figure 2. Histopathologic examination revealed parathyroid adenoma. (A) Endoscopic ultrasound-guided fine-needle aspiration cytology (H\&E, ×400). (B) surgical parathyroidectomy specimen (H\&E, ×400).

went left hemicolectomy, which revealed an organizing abscess and diverticulitis without evidence of malignancy. The patient then underwent right parathyroidectomy, with final diagnosis of parathyroid adenoma (H\&E, $\times 400$ ) (Fig. 2B). His serum calcium and intact PTH levels were normalized after surgery. Informed consent was obtained from the patient.

Parathyroid adenoma is a common cause of hypercalcemia and primary hyperparathyroidism. In cases of deeply situated parathyroid adenomas, US-FNA may fail to target the lesion. In this case, EUS-FNA is a safe and feasible alternative for the diagnosis of ectopic parathyroid adenoma.

\section{Conflict of interest}

No potential conflict of interest relevant to this article was reported. 